\title{
Microarray data analysis to identify crucial genes regulated by CEBPB in human SNB19 glioma cells
}

\author{
Chenghua Du ${ }^{1 *}$, Pan Pan $^{2+}$, Yan Jiang $^{1}$, Qiuli Zhang ${ }^{1}$, Jinsuo Bao ${ }^{1}$ and Chang Liu ${ }^{1}$
}

\begin{abstract}
Background: Glioma is one of the most common primary malignancies in the brain or spine. The transcription factor (TF) CCAAT/enhancer binding protein beta (CEBPB) is important for maintaining the tumor initiating capacity and invasion ability. To investigate the regulation mechanism of CEBPB in glioma, microarray data GSE47352 was analyzed.

Methods: GSE47352 was downloaded from Gene Expression Omnibus, including three samples of SNB19 human glioma cells transduced with non-target control small hairpin RNA (shRNA) lentiviral vectors for $72 \mathrm{~h}$ (normal glioma cells) and three samples of SNB19 human glioma cells transduced with CEBPB shRNA lentiviral vectors for $72 \mathrm{~h}$ (CEBPB-silenced glioma cells). The differentially expressed genes (DEGs) were screened using limma package and then annotated. Afterwards, the Database for Annotation, Visualization, and Integrated Discovery (DAVID) software was applied to perform enrichment analysis for the DEGs. Furthermore, the protein-protein interaction (PPI) network and transcriptional regulatory network were constructed using Cytoscape software.
\end{abstract}

Results: Total 529 DEGs were identified in the normal glioma cells compared with the CEBPB-silenced glioma cells, including 336 up-regulated and 193 down-regulated genes. The significantly enriched pathways included chemokine signaling pathway (which involved CCL2), focal adhesion (which involved THBS1 and THBS2), TGF-beta signaling pathway (which involved THBS1, THBS2, SMAD5, and SMAD6) and chronic myeloid leukemia (which involved TGFBR2 and CCND1). In the PPI network, CCND1 (degree $=29)$ and CCL2 (degree $=12)$ were hub nodes. Additionally, CEBPB and TCF12 might function in glioma through targeting others (CEBPB $\rightarrow$ TCF12, CEBPB $\rightarrow$ TGFBR2, and TCF12 $\rightarrow$ TGFBR2). Conclusions: CEBPB might act in glioma by regulating CCL2, CCND1, THBS1, THBS2, SMAD5, SMAD6, TGFBR2, and TCF12.

Keywords: Glioma, CCAAT/enhancer binding protein beta, Differentially expressed genes, Protein-protein interaction network, Transcriptional regulatory network

\section{Background}

Glioma, which is known as one of the most common primary malignancies in the brain or spine, accounts for nearly $30 \%$ of all brain and central nervous system tumors and $80 \%$ of all malignant brain tumors $[1,2]$. Previous researches have shown that the most important hallmarks of malignant glioma are its invasion and angiogenesis [3]. So far, researchers have indicated that glioma can be induced

\footnotetext{
*Correspondence: ChenghuaDuchd@163.com

${ }^{\dagger}$ Equal contributors

'Department of Neurosurgery, The Affiliated Hospital of Inner Mongolia University for the Nationalities, Huolinhe Street No.1742, Tongliao, Inner Mongolia 028007, China

Full list of author information is available at the end of the article
}

by neurofibromatoses and tuberous sclerosis complex [4], electromagnetic radiation [5], DNA repair genes (such as excision repair cross-complementing 1, ERCC1, and X-ray repair cross-complementing group $1, \mathrm{XRCC1}$ ) [6]. However, the exact molecular mechanisms of glioma were still unclear.

In the central nervous system, the neoplastic transformation can convert the neural cells into cells of mesenchymal phenotype which possess the ability of invasion and promoting angiogenesis $[7,8]$. What is more, it has been identified that mesenchymal stem cells (MSC)-like properties may play a role in the tumorigenesis, invasion, and recurrence of primary glioblastoma tumors [8]. The transcription 
factor (TF) CCAAT/enhancer binding protein beta (CEBPB) is associated with the mesenchymal state of primary glioblastoma, and its expression in glioma is important for maintaining the tumor initiating capacity and invasion ability $[9,10]$. Moreover, the transforming growth factor beta $1 /$ SMAD family member 3 (TGFB1/SMAD3) plays a key role in the extracellular matrix (ECM) production which can lead to glioblastoma aggression $[11,12]$. It has been revealed that $C E B P B$ can regulate the synthesis of ECM [13]. However, the regulation mechanism of CEBPB on TGFB1/ $S M A D 3$ in glioma was seldom studied.

In our study, in order to gain a better understanding of the regulation mechanisms of $C E B P B$ and investigate whether $C E B P B$ could regulate the production of ECM via the TGFB1/SMAD3 signaling pathway in glioma, the microarray data deposited by Carro et al. were further analyzed with bioinformatics methods. Firstly, the differentially expressed genes (DEGs) between SNB19 human glioma cells transduced with non-target control small hairpin RNA (shRNA) lentiviral vectors for $72 \mathrm{~h}$ and SNB19 human glioma cells transduced with $C E B P B$ shRNA lentiviral vectors for $72 \mathrm{~h}$ were identified and annotated. Subsequently, their potential functions were predicted by enrichment analysis. Finally, protein-protein interaction (PPI) network and transcriptional regulatory network were constructed to screen key genes.

\section{Methods}

\section{Microarray dataset}

The microarray dataset of GSE19114 [14] was downloaded from Gene Expression Omnibus (GEO, http:// www.ncbi.nlm.nih.gov/geo/) database, which was based on the platform of GPL6947 IlluminaHumanHT-12 V3.0 expression beadchip. A total of 74 samples were included in the dataset, among which 3 samples of SNB19 human glioma cells transduced with non-target control shRNA lentiviral vectors for $72 \mathrm{~h}$ (normal glioma cells) and 3 samples of SNB19 human glioma cells transduced with $C E B P B$ shRNA lentiviral vectors for $72 \mathrm{~h}(C E B P B-$ silenced glioma cells) were used to study the effect of $C E B P B$ on glioma.

\section{Data preprocessing and DEGs screening}

The preprocessed microarray data were obtained from GEO2R of National Center of Biotechnology Information (NCBI, http://www.ncbi.nlm.nih.gov/geo/geo2r/), including 48803 probes. The linear models for microarray data (limma) package [15] were used to identify the DEGs between the normal glioma cells and the $C E B P B$-silenced glioma cells. Benjamini-Hochberg (BH) method [16] was applied to adjust the raw $p$ value into false discovery rate (FDR). The FDR $<0.05$ and $\mid \log _{2}$ fold change (FC) $>1$ were used as cut-off criteria.

\section{Functional and pathway enrichment analysis}

Gene Ontology (GO, http://www.geneontology.org/) annotations are of great importance for mining biological and functional significance from large dataset [17]. The Kyoto Encyclopedia of Genes and Genomes (KEGG, http://www.genome.ad.jp/kegg) database represents higher order of functions in terms of the network of the interacting molecules [18]. The Database for Annotation, Visualization, and Integrated Discovery (DAVID) online tool [19] was employed to perform GO functional and KEGG pathway enrichment analyses for the DEGs. The $p$ value $<0.05$ was used as the cut-off criterion.

\section{DEGs annotation}

TSGene database (http://bioinfo.mc.vanderbilt.edu/ TSGene/), which contains detailed annotations for each tumor suppressor gene (TSG), such as cancer mutations, gene expressions, methylation sites, transcriptional regulations, and PPIs, was applied to identify the TSGs from the DEGs [20]. Additionally, tumor-associated gene (TAG) database (http://www.binfo.ncku.edu.tw/TAG/), which provides information about commonly shared functional domains in well-characterized oncogenes and TSGs, was used for screening the TAGs from the DEGs [21]. Besides, as a collection of data about the

Table 1 The top ten up- and down-regulated genes

\begin{tabular}{llll}
\hline DEGs & Gene symbol & FDR & Log $_{2}$ FC \\
\hline Up-regulated & AXL & $9.39 \mathrm{E}-07$ & 1.846031 \\
& SERPINE1 & $8.58 \mathrm{E}-07$ & 1.741651 \\
& ITGB1 & $6.28 \mathrm{E}-08$ & 1.739866 \\
& PRPF31 & $6.28 \mathrm{E}-08$ & 1.644503 \\
& TXNDC5 & $3.26 \mathrm{E}-08$ & 1.629988 \\
& WDFY1 & $3.26 \mathrm{E}-08$ & 1.622947 \\
& AXL & $1.57 \mathrm{E}-07$ & 1.554728 \\
& SLC1A3 & $5.96 \mathrm{E}-08$ & 1.484443 \\
& SET & $3.90 \mathrm{E}-07$ & 1.477058 \\
& ITGB1 & $2.66 \mathrm{E}-07$ & 1.466634 \\
AKR1B10 & $3.26 \mathrm{E}-08$ & -2.19537 \\
SLC2A3 & $6.28 \mathrm{E}-08$ & -2.01825 \\
& HMOX1 & $6.28 \mathrm{E}-08$ & -1.58464 \\
CCND1 & $9.30 \mathrm{E}-08$ & -1.49158 \\
HIST1H2BK & $1.16 \mathrm{E}-07$ & -1.38961 \\
STX3 & $3.36 \mathrm{E}-07$ & -1.2468 \\
TDG & $8.98 \mathrm{E}-08$ & -1.23629 \\
SRXN1 & $8.97 \mathrm{E}-07$ & -1.22479 \\
DICER1 & $5.00 \mathrm{E}-07$ & -1.20817 \\
STK40 & $9.14 \mathrm{E}-07$ & -1.19625 \\
\hline
\end{tabular}

DEGs differentially expressed genes, FDR false discovery rate, $F C$ fold change 
transcriptional regulatory network, the Encyclopedia of DNA Elements (ENCODE) project was introduced for screening the TFs from the DEGs [22].

\section{PPI network construction}

The PPI pairs were searched using the Search Tool for the Retrieval of Interacting Genes (STRING, http:// string-db.org/) online tool [23]. The required confidence (combined score) $>0.4$ was used as the cut-off criterion. Then, the Cytoscape software [24] was used to visualize the PPI network. Furthermore, connectivity degree analysis was performed to search the hub nodes of PPI networks. The degree of a node was corresponded to the number of interactions involved it [25]. In addition, hub nodes were nodes with higher degrees.

\section{Transcriptional regulatory network construction}

ENCODE project is a collection of data about the transcriptional regulatory network, which helps illuminate TF-binding sites, histone marks, chromatin accessibility, DNA methylation, RNA expression, RNA binding, and other cell-state indicators [22]. Based on the transcriptional regulation interactions derived from ENCODE project, the regulatory network containing $C E B P B$ and TGFB1/SMAD3 was constructed by Cytoscape software [24].

Table 2 The top ten functions enriched for the differentially expressed genes

\begin{tabular}{|c|c|c|c|c|}
\hline GO ID & Description & Gene number & $p$ value & Gene symbols \\
\hline \multicolumn{5}{|l|}{ (A) } \\
\hline GO:0006366 & Transcription from RNA polymerase II promoter & 47 & $1.01 \mathrm{E}-03$ & $\begin{array}{l}\text { SOX21, TCF25, TOP2A, GTF2F2, CIAO1, } \\
\text { SERPINE1, DKK1, CYR61, SOX18, PAF1... }\end{array}$ \\
\hline GO:0007010 & Cytoskeleton organization & 32 & $2.76 \mathrm{E}-04$ & $\begin{array}{l}\text { PTK2, DPYSL2, CNN3, BICD2, CLIC4, } \\
\text { CTGF, EDN1, NRAS, ITGB1, RHOG... }\end{array}$ \\
\hline GO:0006897 & Endocytosis & 23 & $2.57 \mathrm{E}-05$ & $\begin{array}{l}\text { PTK2, PIK3R2, THBS1, SERPINE1, DKK1, } \\
\text { CYFIP2, AXL, RABEPK, LRPIB, ABCA1... }\end{array}$ \\
\hline GO:0071375 & Cellular response to peptide hormone stimulus & 15 & $5.75 \mathrm{E}-04$ & $\begin{array}{l}\text { PTK2, PIK3R2, GNG10, PPM1A, GNG5, } \\
\text { PIK3R1, ATP6VIG1, NRAS, SOCS2, GNG12.. }\end{array}$ \\
\hline GO:0000398 & mRNA splicing, via spliceosome & 10 & $1.02 \mathrm{E}-02$ & $\begin{array}{l}\text { PABPC1, GTF2F2, LSM7, LSM3, POLR2C, } \\
\text { UPF3B, MBNL2, C1QBP, PRPF31, PAPOLA }\end{array}$ \\
\hline GO:0048469 & Cell maturation & 9 & $8.96 \mathrm{E}-04$ & $\begin{array}{l}\text { SOX18, AXL, GJA1, DLD, FOXO3,TYMS, } \\
\text { CLN5,EPAS1,PTBP3 }\end{array}$ \\
\hline GO:0043200 & Response to amino acid stimulus & 7 & $6.71 \mathrm{E}-04$ & $\begin{array}{l}\text { CTGF, EDN1, CEBPB, TYMS, CCL2, } \\
\text { LAMTOR3, LAMTOR1 }\end{array}$ \\
\hline GO:0006112 & Energy reserve metabolic process & 7 & $4.38 \mathrm{E}-02$ & $\begin{array}{l}\text { GNG10, GNG5, GFPT2, RAP1B, PPP1CC, } \\
\text { GNG12, PYGB }\end{array}$ \\
\hline GO:0018279 & Protein N-linked glycosylation via asparagine & 6 & $1.02 \mathrm{E}-02$ & UGGT1, MLEC, GFPT2, B4GALT5, PGM3, STT3B \\
\hline GO:0006261 & DNA-dependent DNA replication & 6 & $1.49 \mathrm{E}-02$ & POLB, MCM3, RFC5, TOP2A, BAZ1A, RPAIN \\
\hline \multicolumn{5}{|l|}{ (B) } \\
\hline GO:0007167 & Enzyme-linked receptor protein signaling pathway & 19 & $2.89 \mathrm{E}-03$ & $\begin{array}{l}\text { KANK1, RTN4, ATP6V1D, PTPRK, EEF2K, } \\
\text { ERRFI1, CGN, TGFBR2, ATP6VOA1, MVP... }\end{array}$ \\
\hline GO:0043588 & Skin development & 9 & 4.97E-03 & $\begin{array}{l}\text { PTHLH, ALDH3A2, ERRFI1, YAP1, STK4, } \\
\text { EMP1, COL5A2, NCOA3, DICER1 }\end{array}$ \\
\hline GO:0030330 & $\begin{array}{l}\text { DNA damage response, signal transduction by p53 } \\
\text { class mediator }\end{array}$ & 7 & $1.41 \mathrm{E}-04$ & $\begin{array}{l}\text { NDRG1, SPRED1, PSME3, CDKN1A, E2F7, } \\
\text { CASP2, HIPK2 }\end{array}$ \\
\hline GO:0001890 & Placenta development & 7 & 4.74E-04 & TXNRD1, ADM, CCNF, SPP1, STK4, NDP, E2F7 \\
\hline GO:0031100 & Organ regeneration & 5 & $6.05 \mathrm{E}-05$ & ADM, TGFBR2, CCND1, LCP1, CDKN1A \\
\hline GO:0071456 & Cellular response to hypoxia & 5 & $2.26 \mathrm{E}-03$ & HMOX1, NPEPPS, NDRG1, BNIP3, HIPK2 \\
\hline GO:0048002 & Antigen processing and presentation of peptide antigen & 5 & 4.35E-02 & CTSD, NPEPPS, PSME3, AP1S1, AP1S2 \\
\hline GO:0055093 & Response to hyperoxia & 4 & 2.97E-05 & TXNRD1, BNIP3, CAV1, CDKN1A \\
\hline GO:0000188 & Inactivation of MAPK activity & 4 & $1.36 \mathrm{E}-04$ & DUSP5, SPRED1, CAV1, DUSP22 \\
\hline GO:0060443 & Mammary gland morphogenesis & 4 & $2.15 \mathrm{E}-03$ & PTHLH, TGFBR2, CAV1, NCOA3 \\
\hline
\end{tabular}




\section{Results}

\section{Identification of DEGs}

According to the analysis of the microarray dataset, a total of 529 DEGs (including 336 up-regulated genes and 193 down-regulated genes) were identified in the normal glioma cells compared with the $C E B P B$-silenced glioma cells. Among them, the top ten significantly up-regulated genes (such as thrombospondin 1 (THBS1) and chemokine (C-C motif) ligand 2 (CCL2)) and down-regulated genes (such as cyclin D1 (CCND1)) are displayed in Table 1.

\section{Functional and pathway enrichment analysis}

For the up-regulated genes, the enriched functions included transcription from RNA polymerase II promoter

Table 3 The pathways enriched for the differentially expressed genes

\begin{tabular}{|c|c|c|c|c|}
\hline KEGG ID & Name & Gene number & $p$ value & Gene symbols \\
\hline \multicolumn{5}{|l|}{ (A) } \\
\hline 4062 & Chemokine signaling pathway & 12 & 1.63E-03 & $\begin{array}{l}\text { PTK2, PIK3R2, GNG10, GNG5, RAP1B, PIK3R1, NRAS, IL8, } \\
\text { GNG12, CSK, FOXO3, CCL2 }\end{array}$ \\
\hline 4510 & Focal adhesion & 11 & 7.54E-03 & $\begin{array}{l}\text { PTK2, PIK3R2, THBS1, THBS2, RAP1B, PPP1CC, PIK3R1, } \\
\text { ITGB1, ACTG1, FLNB, CAV2 }\end{array}$ \\
\hline 4810 & Regulation of actin cytoskeleton & 11 & 1.18E-02 & $\begin{array}{l}\text { PTK2, PIK3R2, CYFIP2, PPP1CC, PIK3R1, NRAS, ITGB1, } \\
\text { GNG12, ACTG1, CSK, ARHGEF6 }\end{array}$ \\
\hline 4910 & Insulin signaling pathway & 9 & $5.22 \mathrm{E}-03$ & $\begin{array}{l}\text { PIK3R2, PPP1CC, PIK3R1, NRAS, SOCS2, PTPN1, } \\
\text { PYGB, CALM2, PTPRF }\end{array}$ \\
\hline 3013 & RNA transport & 9 & 9.27E-03 & $\begin{array}{l}\text { PABPC1, EIF3A, NUP54, EIF3G, UPF3B, NUP155, } \\
\text { KPNB1, NUP37, EIF2S3 }\end{array}$ \\
\hline 4145 & Phagosome & 8 & 2.82E-02 & $\begin{array}{l}\text { TAP1, THBS1, THBS2, ATP6V1G1, ITGB1, ACTG1, LAMP2, } \\
\text { DYNC1L12 }\end{array}$ \\
\hline 5100 & Bacterial invasion of epithelial cells & 7 & $1.24 \mathrm{E}-03$ & PTK2, PIK3R2, PIK3R1, ITGB1, RHOG, ACTG1, CAV2 \\
\hline 5142 & Chagas disease (American trypanosomiasis) & 7 & 1.13E-02 & PIK3R2, SERPINE1, GNA11, PIK3R1, IL8, IFNGR1, CCL2 \\
\hline 4722 & Neurotrophin signaling pathway & 7 & $3.05 \mathrm{E}-02$ & PIK3R2, RAP1B, PIK3R1, NRAS, CALM2, CSK, FOXO3 \\
\hline 4360 & Axon guidance & 7 & $3.28 \mathrm{E}-02$ & PTK2, DPYSL2, SEMA4F, NRAS, ITGB1, SLIT2, EFNA1 \\
\hline 5131 & Shigellosis & 6 & $3.01 \mathrm{E}-03$ & ITGB1, IL8, RHOG, ACTG1, FBXW11, CSK \\
\hline 5211 & Renal cell carcinoma & 5 & 2.45E-02 & PIK3R2, RAP1B, PIK3R1, NRAS, EPAS1 \\
\hline 5412 & Arrhythmogenic right ventricular cardiomyopathy (ARVC) & 5 & $3.03 \mathrm{E}-02$ & ITGB1, DAG1, GJA1, ACTG1, CDH2 \\
\hline 5410 & Hypertrophic cardiomyopathy & 5 & 4.62E-02 & TPM3, ITGB1, DAG1, TPM1, ACTG1 \\
\hline 4350 & TGF-beta signaling pathway & 5 & 4.83E-02 & THBS1, THBS2, SMAD6, ID3, SMAD5 \\
\hline 20 & Citrate cycle (TCA cycle) & 4 & $5.11 \mathrm{E}-03$ & $C S, D L D, D L A T, S D H A$ \\
\hline 5144 & Malaria & 4 & 3.19E-02 & THBS1, THBS2, IL8, CCL2 \\
\hline 5213 & Endometrial cancer & 4 & 3.39E-02 & PIK3R2, PIK3R1, NRAS, FOXO3 \\
\hline 5223 & Non-small cell lung cancer & 4 & $3.82 \mathrm{E}-02$ & PIK3R2, PIK3R1, NRAS, FOXO3 \\
\hline 3410 & Base excision repair & 3 & 4.23E-02 & POLB, PARP1, PARP3 \\
\hline \multicolumn{5}{|l|}{ (B) } \\
\hline 4144 & Endocytosis & 9 & 4.62E-04 & $\begin{array}{l}\text { ASAP2, VPS36, TGFBR2, ASAP1, CAV1, SH3KBP1, } \\
\text { EHD1, RAB22A, DNM3 }\end{array}$ \\
\hline 4142 & Lysosome & 7 & 4.55E-04 & CTSD, TPP1, ATP6VOA1, ABCB9, AP1S1, AP1S2, NEU1 \\
\hline 2010 & $\mathrm{ABC}$ transporters & 4 & $1.58 \mathrm{E}-03$ & $A B C C 2, A B C C 3, A B C B 9, A B C C 5$ \\
\hline 10 & Glycolysis/gluconeogenesis & 4 & $6.56 \mathrm{E}-03$ & ENO2, ALDH3A2, PGAM1, PGK1 \\
\hline 5220 & Chronic myeloid leukemia & 4 & 9.85E-03 & TGFBR2, CCND1, CDKN1A, BCL2L1 \\
\hline 561 & Glycerolipid metabolism & 3 & 1.98E-02 & ALDH3A2, AGPAT9, LCLAT1 \\
\hline 5212 & Pancreatic cancer & 3 & 4.69E-02 & TGFBR2, CCND1, BCL2L1 \\
\hline 4966 & Collecting duct acid secretion & 2 & 3.85E-02 & ATP6V1D, ATP6VOA1 \\
\hline 650 & Butanoate metabolism & 2 & 4.67E-02 & AKR1B10, HMGCS1 \\
\hline
\end{tabular}

(A) The pathways enriched for the up-regulated genes. (B) The pathways enriched for the down-regulated genes. Kyoto Encyclopedia of Genes and Genomes, KEGG; identification, ID 
( $p=1.01 \mathrm{E}-03)$, cytoskeleton organization $(p=2.76 \mathrm{E}-04)$, and endocytosis $(p=2.57 \mathrm{E}-05)$ (Table 2A). Meanwhile, the down-regulated genes were mainly enriched in the function of enzyme-linked receptor protein signaling pathway ( $p=2.89 \mathrm{E}-03)$, skin development $(p=4.97 \mathrm{E}-03)$, and response to hyperoxia $(p=2.97 \mathrm{E}-05)$ (Table $2 \mathrm{~B})$.

Among the up-regulated genes, CCL2 was significantly enriched in the pathway of chemokine signaling pathway $(p=1.63 \mathrm{E}-03) . T H B S 1$ and thrombospondin 2 (THBS2) were significantly involved in the pathway of focal adhesion $(p=7.54 \mathrm{E}-03)$. And the up-regulated genes, such as THBS1, THBS2, SMAD family member 5 (SMAD5) and SMAD family member 6 (SMAD6), were significantly enriched in transforming growth factor beta (TGF-beta) signaling pathway $(p=4.83 \mathrm{E}-02)$ (Table $3 \mathrm{~A})$. Meanwhile, the down-regulated transforming growth factor beta receptor II (TGFBR2) and CCND1 were significantly enriched in both the pathways of chronic myeloid leukemia $(p=9.85 \mathrm{E}$ $-03)$ and pancreatic cancer $(p=4.69 \mathrm{E}-02)$ (Table 3B).

\section{The annotation of DEGs}

A total of 54 DEGs were screened as TAGs, including 33 up-regulated and 21 down-regulated genes. Among the 33 up-regulated genes, there were 22 TSGs (such as THBS1), 6 oncogenes, and 5 other genes (such as CCL2). Meanwhile, there were 13 TSGs, 4 oncogenes (such as CCND1), and 4 other genes in the 21 downregulated genes. Additionally, 9 DEGs were screened as the TFs, including 8 up-regulated and 1 down-regulated genes (Table 4).

\section{PPI network analysis}

The constructed PPI network was consisted of 810 interactions (such as CCND1-THBS1 and THBS1-CCL2) (Fig. 1). Besides, the top $10 \%$ nodes with higher degrees in the PPI network were identified, including CCND1 $($ degree $=29)$ and CCL2 $($ degree $=12)($ Table 5$)$.

\section{Transcriptional regulatory network analysis}

For further study, the regulation of TGFB1/SMAD3 by $C E B P B$, the transcriptional regulation interactions related to TGFB1/SMAD3, and the members of TGFB family were screened out from the ENCODE database and the transcriptional regulatory network was visualized by Cytoscape software (Fig. 2). The transcriptional regulation network showed that the $C E B P B$ could regulate $S M A D 3$, transcription factor 12 (TCF12), transforming growth factor beta 2 (TGFB2), TGFBR2, and TGFBR3 directly. Additionally, TCF12 targeted TGFB1, TGFBR1, TGFBR2, TGFBR3, and SMAD3.

\section{Discussion}

In this study, a total of 529 DEGs were obtained, including 336 up-regulated genes and 193 down-regulated genes. Enrichment analysis indicated that the up-regulated CCL2 was significantly enriched in the chemokine signaling pathway. Reports have found that chemokine expressed by stromal cells or endogenously produced in glioma cells may play key roles in tumor cell migration, invasion, proliferation, angiogenesis and immune cell infiltration in the tumor mass [26]. The chemokine CCL2 can promote glioma tumor aggressiveness by promoting attraction of $\mathrm{T}$ regulatory cells (which suppress the lymphocyte anti-tumor effector function) and microglial cells (which can reduce the anti-tumor functions and secrete pro-invasive metalloproteinases) [27, 28]. Meanwhile, metalloproteinases can promote the glioma invasion through the detachment of ECM [29]. Besides, results of DEGs annotation showed that CCL2 was screened out as a TAG. Therefore, we speculated that the increased expression of CCL2 could promote glioma aggressiveness through the pathway of chemokine signaling.

In addition, some up-regulated genes (such as THBS1, THBS2, SMAD5, and SMAD6) were significantly enriched in the TGF-beta signaling pathway in our study. Recently, it has been reported that the TGFB is a key factor in controlling migration, invasion and angiogenesis in glioblastoma and induces profound immunosuppression [30]. Besides, the THBS1 (belonging to thrombospondin family), which is referred as a TGFB activating protein, induces the glioma invasion [31]. THBS1 is a powerful antiangiogenesis protein in glioblastoma [32]. These suggested that THBS1 might play a key role in regulating the

Table 4 The identified transcription factors (TFs) and tumor associated genes (TAGs) among the differentially expressed genes (DEGs). Tumor suppressed genes, TSGs

\begin{tabular}{|c|c|c|c|c|c|c|}
\hline \multirow[t]{2}{*}{ DEGs } & \multirow{2}{*}{$\begin{array}{l}\text { TF } \\
\text { numbers }\end{array}$} & \multirow[t]{2}{*}{ TFs } & \multirow{2}{*}{$\begin{array}{l}\text { TAG } \\
\text { numbers }\end{array}$} & \multicolumn{3}{|l|}{ TAGs } \\
\hline & & & & TSGs & Oncogenes & Others \\
\hline Up-regulated & 1 & KLF12 & 33 & $\begin{array}{l}\text { BAP1, THBS1, DKK1, PAF1, ST13, } \\
\text { LRP1B, PDGFRL, ITGB1, TPM1, } \\
\text { GJA1, CDH11, SLIT2, GLIPR1, } \\
\text { FAT1, SOD2, FOXO3, EFNA1, GAS1, } \\
\text { PTPRF, RAD51C, CAV2, SDHA }\end{array}$ & $\begin{array}{l}\text { SET, CCNA2, AXL, } \\
\text { NRAS, ROS1, SCK }\end{array}$ & $\begin{array}{l}\text { GTF2F2, CTGF, FHL2, } \\
\text { C1QBP, CCL2 }\end{array}$ \\
\hline Down-regulated & 8 & $\begin{array}{l}\text { ASCL1, ETV4, HSF1, LMO3, } \\
\text { PML, RUNX3, TCF7, USF2 }\end{array}$ & 21 & $\begin{array}{l}\text { HIPK2, YAP1, ERRFI1, PTPRK, KANK1, } \\
\text { BNIP3L, DUSP22, SASH1, CDKN1A, } \\
\text { NDRG4, ZFHX3, NDRG1, TGFBR2, }\end{array}$ & $\begin{array}{l}\text { BCL2L2, NCOA3, } \\
\text { CCND1, CDC25B }\end{array}$ & PTHLH, EMP1, CAV1, GLS \\
\hline
\end{tabular}




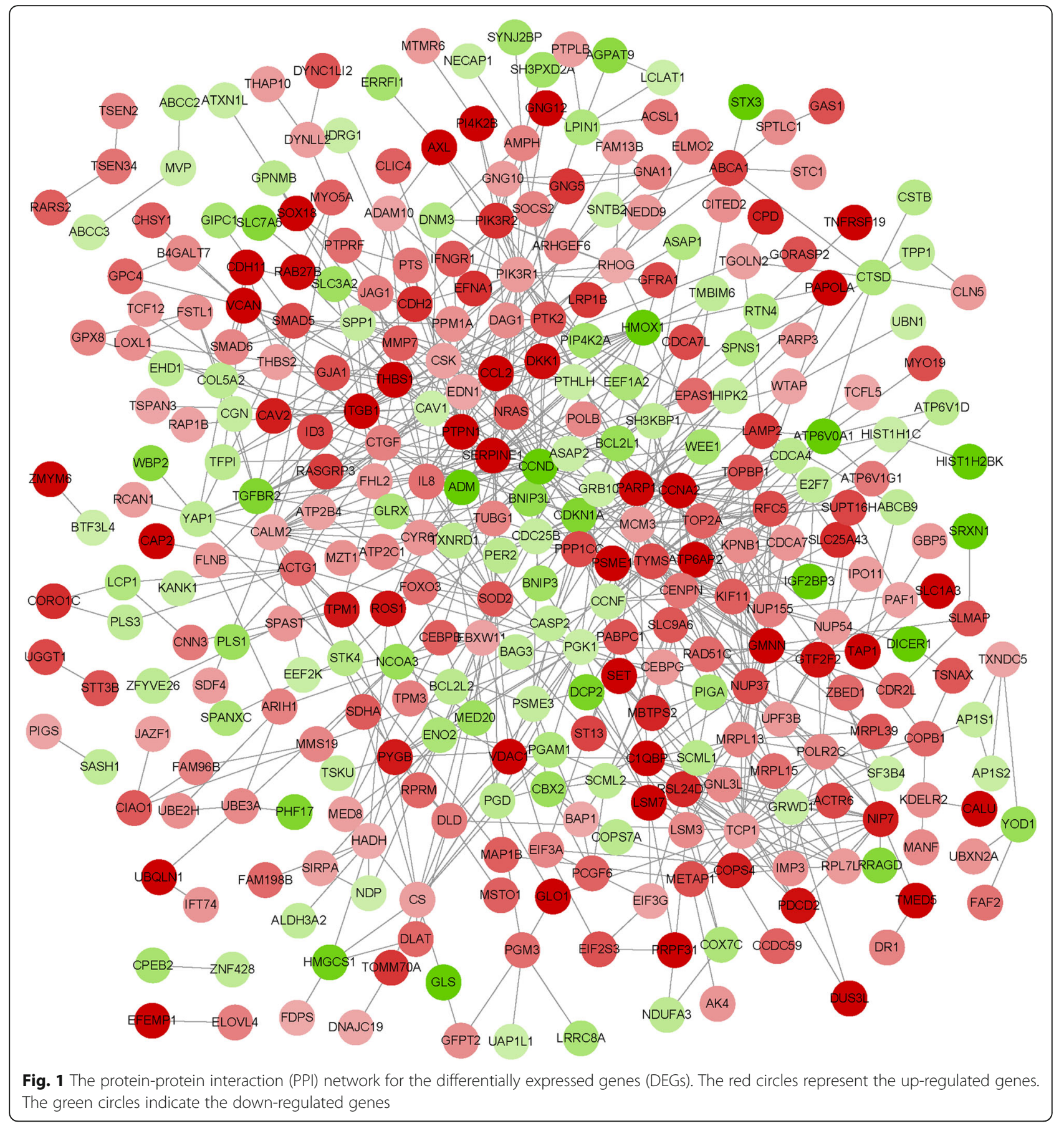

angiogenesis in glioma. As another member of thrombospondin family, THBS2 may be a potential inhibitor of tumor growth and angiogenesis [33]. Moreover, it has been shown that THBS2 can function as an endogenous inhibitor of angiogenesis through directly affecting endothelial cell migration, proliferation, survival, and apoptosis [34]. In our study, we also found that THBS1 and THBS2 were significantly involved in the pathway of focal adhesion. Previous study reported that focal adhesion can suppress the migration and metastasis of tumor cells [35].
Therefore, we speculated that THBS1 and THBS2 could regulate angiogenesis and invasion in glioma via TGF-beta signaling pathway and focal adhesion pathway. Former researches have shown that SMAD6 is an inhibitor of TGFB signaling and blocked the phosphorylation of receptorregulated SMADs (such as SMAD5) in the cytoplasm [36]. As a result, we assumed that SMAD5 and SMAD6 might affect glioma by regulating the TGFB signaling. In the PPI network, THBS1 could interact with CCL2, to some extent, indicating that THBS1 might play key roles in glioma 
Table 5 The top $10 \%$ DEGs with higher degrees in the protein-protein interaction (PPI) network

\begin{tabular}{|c|c|c|c|c|c|c|c|}
\hline Gene & Degree & Gene & Degree & Gene & Degree & Gene & Degree \\
\hline$\overline{C C N D 1}$ & 29 & SOD2 & 19 & CENPN & 16 & KIF11 & 15 \\
\hline PIK3R1 & 25 & TYMS & 19 & CAV1 & 16 & PTK2 & 15 \\
\hline$P G K 1$ & 22 & CDKNIA & 18 & PIK3R2 & 16 & EDN1 & 14 \\
\hline NUP37 & 22 & PARP1 & 18 & CTGF & 15 & CS & 13 \\
\hline CALM2 & 21 & $T O P 2 A$ & 18 & RFC5 & 15 & CCL2 & 13 \\
\hline MCM3 & 21 & $\mid T G B\rceil$ & 18 & NUP155 & 15 & $R S L 24 D 1$ & 12 \\
\hline GMNN & 20 & TCP1 & 18 & NRAS & 15 & CDCA7 & 12 \\
\hline CCNA2 & 20 & SERPINEI & 17 & NIPT & 15 & $B C L 2 L 1$ & 12 \\
\hline
\end{tabular}

through regulating CCL2. Consequently, THBS1, THBS2, $S A M D 5$ and SMAD6 could be key factors involved in the $C E B P B$-silenced glioma.

Moreover, CCND1, as a member of the cyclin family, possessed the highest degree in the PPI network. Cyclins can modulate tumor cell cycle through alterations in cyclin-dependent kinase activity [37]. What's more, researchers have discovered that overexpression of CCND1 can elevate the proliferation and invasion potential of human glioblastoma cells [38]. In the PPI network, we also found that CCND1 had interaction with THBS1, suggesting that CCND1 could be involved in regulating proliferation and invasion of glioma via interacting with THBS1.

TGFBR2 plays a key role in TGFB signal propagation via activating TGFBR1 and the phosphorylation of SMAD proteins [39]. Moreover, silencing of TGFBR2 can abolish TGFB-induced invasion and migratory responses of glioblastoma in vitro [40]. In our study, we also discovered that the up-regulated TCF12 could regulate TGFB1 and SMAD3, indicating that $C E B P B$ might regulate TGFB1 and SMAD3 through TCF12. Previous studies have shown that TGFB1/
SMAD3 can promote tumor cell migration, invasion and metastasis through inducing epithelial-mesenchymal transition $[41,42]$. What is more, TCF12 has been found to suppress the expression of E-cadherin, which can lead to the metastasis of tumor cells [43]. Therefore, we assumed that $C E B P B$ might regulate TGFBR2 and SMAD3 through TGF$\beta 1 / S M A D 3$ signaling pathway in glioma, and $C E B P B$ could also affect metastasis of glioma by regulating TCF12. However, in our study, TGFB1 and SMAD3 were not significantly expressed, which might due to the relatively short time for $C E B P B$ silencing. In our further research, the regulation of $C E B P B$ on TGFB1/SMAD3 will be studied with $C E B P B$-silenced for a relatively long time.

\section{Conclusions}

We conducted a comprehensive bioinformatics analysis to identify genes which may be correlated with $C E B P B$-silenced glioma. A total of 529 DEGs were identified in the normal glioma cells compared with the CEBPB-silenced glioma cells. Besides, The identified DEGs, such as TCF12, TGFBR2, CCL2, THBS1, THBS2, SMAD5, SMAD6, and $C C N D 1$, might play important roles in the progression of

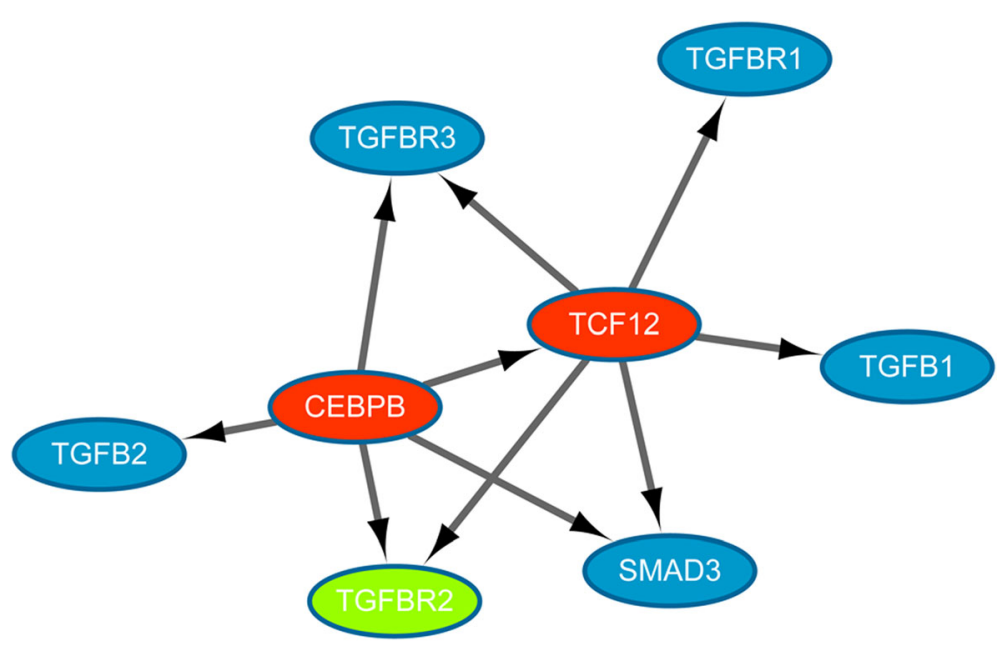

Fig. 2 The transcriptional regulatory network involving CEBPB and TGFB1/SMAD3. The red and green nodes represent the up-regulated and down-regulated genes, respectively. The blue nodes stand for non-differentially expressed genes (DEGs). The arrows represent regulatory relationships 
glioma via the regulation of $C E B P B$. However, further researches are still needed to unravel their action mechanisms in glioma.

\section{Abbreviations}

BH: Benjamini-Hochberg; DEGs: Differentially expressed genes; ECM: Extracellular matrix; ENCODE: Encyclopedia of DNA Elements; FDR: False discovery rate; GEO: Gene Expression Omnibus; KEGG: The Kyoto Encyclopedia of Genes and Genomes; MSC: Mesenchymal stem cells; PPI: Protein-protein interaction; TAG: Tumor-associated gene; TF: Transcription factor; TSG: Tumor suppressor gene

\section{Acknowledgements}

None.

\section{Funding}

None.

\section{Availability of data and materials}

The datasets supporting the conclusions of this article are too many to share. There was no new software.

\section{Authors' contribution}

CHD and PP participated in the design of this study, and they both performed the statistical analysis. PP, YJ, QZ, JSB, and CL carried out the study and collected important background information. CHD and PP drafted the manuscript. All authors read and approved the final manuscript.

\section{Competing interests}

The authors declare that they have no competing interests.

\section{Consent for publication}

Not applicable.

\section{Ethics approval and consent to participate}

Not applicable.

\section{Author details}

'Department of Neurosurgery, The Affiliated Hospital of Inner Mongolia University for the Nationalities, Huolinhe Street No.1742, Tongliao, Inner Mongolia 028007, China. ${ }^{2}$ Department of Hepatology, Tongliao City Hospital for Infectious Diseases, Tongliao, Inner Mongolia 028007, China.

Received: 7 June 2016 Accepted: 30 August 2016

Published online: 06 October 2016

\section{References}

1. Mei P, Bai J, Shi M, Liu Q, Li Z, Fan Y, Zheng J. BRMS1 suppresses glioma progression by regulating invasion. Migration and adhesion of glioma cells. PLoS One. 2014:9:e98544.

2. Goodenberger ML, Jenkins RB. Genetics of adult glioma. Cancer Genet. 2012;205:613-21.

3. Onishi M, Ichikawa T, Kurozumi K, Date I. Angiogenesis and invasion in glioma. Brain Tumor Pathol. 2011;28:13-24

4. Reuss D, von Deimling A. Hereditary tumor syndromes and gliomas. In: Gliomas. Heidelberg: Springer Berlin; 2009. p. 83-102.

5. Cancer IAfRo. IARC classifies radiofrequency electromagnetic fields as possibly carcinogenic to humans. Lyon: World Health Organization; 2011

6. Fahmideh MA, Schwartzbaum J, Frumento P, Feychting M. Association between DNA repair gene polymorphisms and risk of glioma: a systematic review and meta-analysis. Neuro Oncol. 2014;16:807-14.

7. Phillips HS, Kharbanda S, Chen R, Forrest WF, Soriano RH, Wu TD, Misra A, Nigro JM, Colman H, Soroceanu L. Molecular subclasses of high-grade glioma predict prognosis, delineate a pattern of disease progression, and resemble stages in neurogenesis. Cancer Cell. 2006;9:157-73.

8. Tso C-L, Shintaku P, Chen J, Liu Q, Liu J, Chen Z, Yoshimoto K, Mischel PS, Cloughesy TF, Liau LM. Primary glioblastomas express mesenchymal stem-like properties. Mol Cancer Res. 2006:4:607-19.

9. Singh R, Sharma MC, Sarkar C, Singh M, Chauhan SS. Transcription factor C/EBP- $\beta$ mediates downregulation of dipeptidyl-peptidase III expression by interleukin-6 in human glioblastoma cells. FEBS J. 2014;281:1629-41.
10. Aguilarmorante D, Moralesgarcia JA, Santos A, Perezcastillo A. CCAAT/ enhancer binding protein $\beta$ induces motility and invasion of glioblastoma cells through transcriptional regulation of the calcium binding protein S100A4. Oncotarget. 2015:6:454-63.

11. Okano K, Hibi A, Miyaoka T, Inoue T, Sugimoto H, Tsuchiya K, Akiba T, Nitta K. Inhibitory effects of the transcription factor Ets-1 on the expression of type I collagen in TGF- $\beta 1$-stimulated renal epithelial cells. Mol Cell Biochem. 2012;369:247-54.

12. Barnes JM, Weaver VM. Abstract B04: the role of mechanical force and integrin-ECM signaling in glioblastoma aggression. Cancer Res. 2013;73:B04.

13. Luft FC. C/EBP $\beta$ LIP induces a tumor menagerie making it an oncogene. J Mol Med. 2015;93:1-3.

14. Carro MS, Lim WK, Alvarez MJ, Bollo RJ, Zhao X, Snyder EY, Sulman EP, Anne SL, Doetsch F, Colman H. The transcriptional network for mesenchymal transformation of brain tumours. Nature. 2009:463:318-25.

15. Ritchie ME, Phipson B, Wu D, Hu Y, Law CW, Shi W, Smyth GK. Limma powers differential expression analyses for RNA-sequencing and microarray studies. Nucleic Acids Res. 2015;43:e47.

16. Ghosh D. Incorporating the empirical null hypothesis into the BenjaminiHochberg procedure. Stat Appl Genet Mol Biol. 2012;11:1-21.

17. Consortium GO. Gene ontology consortium: going forward. Nucleic Acids Res. 2015:43:1049-56.

18. Du J, Yuan Z, Ma Z, Song J, Xie X, Chen Y. KEGG-PATH: Kyoto encyclopedia of genes and genomes-based pathway analysis using a path analysis model. Mol Biosyst. 2014;10:2441-7.

19. Jiao X, Sherman BT, Huang DW, Stephens R, Baseler MW, Lane HC, Lempicki RA. DAVID-WS: a stateful web service to facilitate gene/protein list analysis. Bioinformatics. 2012;28:1805-6.

20. Zhao M, Sun J, Zhao Z. TSGene: a web resource for tumor suppressor genes. Nucleic Acids Res. 2013;41:D970-6.

21. Chen J-S, Hung W-S, Chan H-H, Tsai S-J, Sun HS. In silico identification of oncogenic potential of fyn-related kinase in hepatocellular carcinoma. Bioinformatics. 2013;29:420-7.

22. Raney BJ, Cline MS, Rosenbloom KR, Dreszer TR, Learned K, Barber GP, Meyer LR, Sloan CA, Malladi VS, Roskin KM, et al. ENCODE whole-genome data in the UCSC genome browser (2011 update). Nucleic Acids Res. 2011;39:30.

23. Franceschini A, Szklarczyk D, Frankild S, Kuhn M, Simonovic M, Roth A, Lin J, Minguez $P$, Bork $P$, von Mering C. STRING v9. 1: protein-protein interaction networks, with increased coverage and integration. Nucleic Acids Res. 2013:41:D808-15.

24. Saito R, Smoot ME, Ono K, Ruscheinski J, Wang P-L, Lotia S, Pico AR, Bader GD, Ideker T. A travel guide to Cytoscape plugins. Nat Methods. 2012:9:1069-76.

25. Chao W, Zhu J, Zhang X. Integrating gene expression and protein-protein interaction network to prioritize cancer-associated genes. BMC Bioinf. 2012;13:1-10

26. Domanska UM, Kruizinga RC, Dunnen WFAD, Timmer-Bosscha H, Vries EGED, Walenkamp AME. The chemokine network, a newly discovered target in high grade gliomas. Crit Rev Oncol Hematol. 2011;79:154-63.

27. Carrillo-de Sauvage MA, Gómez A, Ros CM, Ros-Bernal F, Martín ED, PerezVallés A, Gallego-Sanchez JM, Fernández-Villalba E, Sr BC, Jr BC. CCL2expressing astrocytes mediate the extravasation of $\mathrm{T}$ lymphocytes in the brain. Evidence from patients with glioma and experimental models in vivo. PLoS One. 2012;7:e30762

28. Lindemann C, Marschall V, Weigert A, Klingebiel T, Fulda S. Smac mimeticinduced upregulation of CCL2/MCP-1 triggers migration and invasion of glioblastoma cells and influences the tumor microenvironment in a paracrine manner 1. Neoplasia. 2015;17:481-9.

29. Könnecke $H$, Bechmann I. The role of microglia and matrix metalloproteinases involvement in neuroinflammation and gliomas. Clin Dev Immunol. 2013:2013:914104.

30. Roth P, Silginer M, Goodman SL, Hasenbach K, Thies S, Maurer G, Schraml P, Tabatabai G, Moch H, Tritschler I. Integrin control of the transforming growth factor- $\beta$ pathway in glioblastoma. Brain. 2013;136:564-76.

31. Seliger C, Leukel P, Moeckel S, Jachnik B, Lottaz C, Kreutz M, Brawanski A, Proescholdt M, Bogdahn U, Bosserhoff A-K. Lactate-modulated induction of THBS-1 activates transforming growth factor (TGF)-beta2 and migration of glioma cells in vitro. PLoS One. 2013:8:e78935.

32. Brooks MD, Jackson E, Piwnica-Worms D, Mitre RD, Rubin JB. Downregulation of THBS1 is a critical step in glioblastoma angiogenesis. In: Cancer research Philadelphia: AMER Assoc Cancer Research; 2013. 
33. Hawighorst T, Velasco P, Streit M, Hong YK, Kyriakides TR, Brown LF, Bornstein P, Detmar M. Thrombospondin-2 plays a protective role in multistep carcinogenesis: a novel host anti-tumor defense mechanism. EMBO J. 2001:20:2631-40.

34. Lawler PR, Lawler J. Molecular basis for the regulation of angiogenesis by thrombospondin-1 and -2. Cold Spring Harb Perspect Med. 2012;2:a006627.

35. Jarjour AA, Durko M, Luk TL, Marçal N, Shekarabi M, Kennedy TE. Autocrine netrin function inhibits glioma cell motility and promotes focal adhesion formation. PLoS One. 2011;6:e25408.

36. Jung SM, Lee JH, Park J, Oh YS, Lee SK, Park JS, Lee YS, Kim JH, Lee JY, Bae YS, et al. Smad6 inhibits non-canonical TGF- $\beta 1$ signalling by recruiting the deubiquitinase A20 to TRAF6. Nat Commun. 2013;4:2562.

37. Casimiro MC, Crosariol M, Loro E, Li Z, Pestell RG. Cyclins and cell cycle control in cancer and disease. Genes Cancer. 2012;3:649-57.

38. Phull P, Shipley A, Mowat N. NOTCH3 is a prognostic factor that promotes glioma cell proliferation, migration and invasion via activation of CCND1 and EGFR. PLoS One. 2013;8:271-2.

39. Rožmarić M, IVšić AG, Grahek Ž. TGF-beta induced Erk phosphorylation of smad linker region regulates smad signaling. PLoS One. 2012;7:2016.

40. Wesolowska A, Kwiatkowska A, Slomnicki L, Dembinski M, Master A, Sliwa M, Franciszkiewicz K, Chouaib S, Kaminska B. Microglia-derived TGF- $\beta$ as an important regulator of glioblastoma invasion - an inhibition of TGF- $\beta$ dependent effects by shRNA against human TGF- $\beta$ type II receptor. Oncogene. 2007;27:918-30.

41. Fuxe J, Vincent T, Garcia de Herreros A. Transcriptional crosstalk between TGF-beta and stem cell pathways in tumor cell invasion: role of EMT promoting Smad complexes. Cell Cycle. 2010;9:2363-74.

42. Bae E, Kim S-J, Hong S, Liu F, Ooshima A. Smad3 linker phosphorylation attenuates Smad3 transcriptional activity and TGF- $\beta 1 /$ Smad3-induced epithelial-mesenchymal transition in renal epithelial cells. Biochem Biophys Res Commun. 2012:427:593-9.

43. Lee CC, Chen WS, Chen CC, Chen LL, Lin YS, Fan CS, Huang TS. TCF12 protein functions as transcriptional repressor of $\mathrm{E}$-cadherin, and its overexpression is correlated with metastasis of colorectal cancer. J Biol Chem. 2011;287:2798-809.

\section{Submit your next manuscript to BioMed Central and we will help you at every step:}

- We accept pre-submission inquiries

- Our selector tool helps you to find the most relevant journal

- We provide round the clock customer support

- Convenient online submission

- Thorough peer review

- Inclusion in PubMed and all major indexing services

- Maximum visibility for your research

Submit your manuscript at www.biomedcentral.com/submit

) Biomed Central 